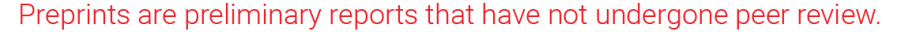 They should not be considered conclusive, used to inform clinical practice, or referenced by the media as validated information. \\ High Expression of PXN Predicts a Poor Prognosis in Acute myeloid leukemia
}

\section{xinwen zhang ( $564388430 @ q q . c o m$ )}

The Affiliated Hospital of Southwest Medical University https://orcid.org/0000-0002-7424-4900

\section{Hao Xiong}

The Affiliated Hospital of Southwest Medical University

Jialin Duan

The Affiliated Hospital of Southwest Medical University

\section{Xiaomin Chen}

The Affiliated Hospital of Southwest Medical University

\section{Yang Liu}

The Affiliated Hospital of Southwest Medical University

\section{Chunlan Huang}

The Affiliated Hospital of Southwest Medical University https://orcid.org/0000-0003-1280-9950

\section{Research Article}

Keywords: Acute myeloid leukemia, PXN, prognosis, Paxillin, overall survival

Posted Date: September 3rd, 2021

DOI: https://doi.org/10.21203/rs.3.rs-847908/v1

License: (c) (i) This work is licensed under a Creative Commons Attribution 4.0 International License.

Read Full License 


\title{
High Expression of PXN Predicts a Poor Prognosis in Acute myeloid leukemia
}

Xinwen Zhang ${ }^{1 \#}$, Hao Xiong ${ }^{1 \#}$, Jialin Duan ${ }^{1}$, Xiaomin Chen ${ }^{1}$, Yang Liu ${ }^{1}$, Chunlan Huang ${ }^{1 *}$

Affiliations

${ }^{I}$ Stem cell laboratory and Department of Hematology, Affiliated hospital of southwest medical university, Luzhou city, Sichuan province, China

*Corresponding author: Chunlan Huang

Stem cell laboratory and Department of Hematology, Affiliated hospital of southwest medical university, Luzhou city, Sichuan province, China

Tel: +8618090859929

Postal code: 646000

Email: huangchunlan@swmu.edu.cn

ORCID ID : https://orcid.org/0000-0003-1280-9950

\begin{abstract}
Background: Acute myeloid leukemia (AML) is one of the common malignant diseases of hematopoietic system. Paxillin $(P X N)$ is an important part of focal adhesions (FAs), which is related to the poor prognosis of many kinds of malignant tumors. However, no research has focused on the expression of PXN in AML. We aimed to investigate the expression of PXN in AML and its prognostic significance.
\end{abstract}

Methods: Using GEPIA and UALCAN database to analyze the expression of $P X N$ in AML patients and its prognostic significance. Bone marrow samples of newly diagnosed AML patients were collected to extract RNA, and qRT-PCR was used to detect the expression of $P X N$. The 
prognosis was followed up. Chi-square test was used to analyze the relationship between $P X N$ expression and clinical laboratory characteristics. Kaplan-Meier analysis was used to draw survival curve, and Cox regression analysis was used to analyze the independent factors affecting the prognosis of patients with AML. The co-expression genes of $P X N$ were analyzed by LinkedOmics to explore its biological significance in AML.

Results: Kaplan-Meier analysis showed that the overall survival time of AML patients was related to whether to receive treatment and $P X N$ expression $(\mathrm{P}<0.05)$. COX regression analysis showed that whether to receive treatment $(\mathrm{HR}=0.227,95 \% \mathrm{CI}=0.075-0.689, P=0.009)$ and high expression of $P X N(\mathrm{HR}=4.484,95 \% \mathrm{CI}=1.449-13.889, P=0.009)$ were independent poor prognostic factors in patients with AML.

Conclusion: $P X N$ is highly expressed in AML patient, and high $P X N$ expression is an indicator of poor prognosis in AML patient.

Keywords: Acute myeloid leukemia, $P X N$, prognosis, Paxillin, overall survival

\section{Introduction}

Acute myeloid leukemia (acute myeloid leukemia, AML) is a kind of hematopoietic malignant tumor with high heterogeneity, which is characterized by excessive proliferation of bone marrow primordial myeloid cells, accounting for about $60 \%$ of adult acute leukemia[1]. Now it is generally believed that the biological characteristics of AML are caused and determined by its own genetic abnormalities[2]. According to the effect of cytogenetic differences on prognosis, AML can be divided into low-risk group, medium-risk group and high-risk group. The five-year overall survival rate (overall survival OS) is $55 \%, 24 \%$ and $5 \%$, respectively [3]. However, due to the limitation of methodological sensitivity, nearly half of AML patients still fail to detect genetic abnormalities using existing cytogenetic detection techniques[4]. Therefore, it is extremely important to look for abnormal molecules related to the prognosis of AML, classify the risk of patients, and then adopt stratified treatment to improve the therapeutic effect.

The paxillin $(P X N)$ gene is located on human chromosome 12q24.31 and encodes for a focal adhesion molecule of $68 \mathrm{kDa}$, which is a multidomain adaptor protein connecting extracellular matrices to the cytoskeleton[5, 6]. Paxillin plays an important role in the process of transforming 
extracellular signal into intracellular biological response, which is triggered by the binding of integrin and extracellular matrix[7]. $P X N$ is generally considered to be related to the movement, migration and invasion of cancer cells, and ultimately related to tumor metastasis. Studies have shown that $P X N$ functional mutations are associated with the malignant progression of many tumors, including breast cancer, lung carcinoma[5], prostate cancer[8], melanoma and colorectal cancer[9]. To the best of our knowledge, the function of $P X N$ has not been reported in AML. In this study, we focused on the expression and prognostic significance of $P X N$ via bioinformatics analysis and detection of expression in clinical samples.

\section{Methods}

\subsection{Data Source}

GEPIA (http://gepia.cancer-pku.cn/) is a web-based tool based on The Cancer Genome Atlas (TCGA) and Genotype-Tissue Expression (GTEx) project. It provides rapid and customizable functions and key interactive functions, including differential expression analysis, profiling plotting, correlation analysis and patient survival analysis[10]. UALCAN (http://ualcan.path.uab.edu) is an easy-to-use interactive portal for in-depth analysis of TCGA gene expression data[11]. In our study, we studied the prognostic value of $P X N$ gene in the "Single Gene Analysis" module of GEPIA database. UALCAN was used to verify the prognostic value of $P X N$ through the "Survival Analysis" module and the "AML" dataset. Expression data of $P X N$ was examined via the "Expression Analysis" module and the "AML" dataset. The P value cutoff was 0.05 . The LinkedOmics (http://www.linkedomics.org/login. php) is an interactive web-portal including 32 TCGA cancerassociated data[12]. We used Pearson test to analyze the expression of differential genes related to $P X N$ in AML, and GESA was used to analyze GO-BP and KEGG pathways. The threshold was based on the following values: FDR of 0.05 and simulations of 500 .

\subsection{Patients}

We collected bone marrow blood from 27 newly diagnosed AML patients in the affiliated Hospital of Southwest Medical University from December 2019 to March 2021. The study was approved by the Clinical trial Ethics Committee of the affiliated Hospital of Southwest Medical University in accordance with Helsinki guidelines for the protection of human subjects. All 
participants freely gave written informed consent. The diagnosis of AML was based on the 2008 WHO criteria [13]. The exclusion criteria for selected patients with AML are as follows: (1) relapsed AML, (2) with other malignant tumors, (3) with severe hepatic and renal insufficiency, (4) received hematopoietic stem cell transplantation. All the patients were mainly treated with DA (daunorubicin $45 \mathrm{mg} / \mathrm{m} 2 /$ day for 3 days and cytarabine $100 \mathrm{mg} / \mathrm{m} 2 /$ day for 7 days) and IA regimen (idarubicin $8-10 \mathrm{mg} / \mathrm{m} 2 /$ day for 3 days and cytarabine $150 \mathrm{mg} / \mathrm{m} 2 /$ day for 7 days).

\subsection{Quantitative real-time PCR analysis (qRT-PCR)}

Fresh bone marrow samples were collected. Total RNA was extracted from fresh bone marrow samples using the TRIzol Reagent (TIANGEN) according to the manufacturers' protocol. RNA was Reverse-transcribed using a Rever Tra Ace qPCR RT Kit (Toyobo). The concentration and quality of RNA samples were examined by Nanodrop (Thermo Fisher).RT-qPCR was conducted using a Light Cycler 96 (Roche).with the following amplification protocol: denaturation at $95^{\circ} \mathrm{C}$ for $30 \mathrm{~s}$, 40 cycles of denaturation at $95^{\circ} \mathrm{C}$ for $5 \mathrm{~s}$, annealing at $55^{\circ} \mathrm{C}$ for $10 \mathrm{~s}$, and extension at $72^{\circ} \mathrm{C}$ for $15 \mathrm{~s}$. The mRNA expression levels were normalized to GAPDH. The sequences of primers were as follows: $\quad P X N \quad$ 5'-GATTCATCCACCAGCAGCCTCAG-3'(forward) and 5'GCACGGAGAGCCAACACTGTC-3'(reverse); All primers were synthesized by Sangon Biotech (Shanghai, China). Relative messenger RNA (mRNA) levels were measured using the $2^{\text {-Acycle threshold }}$ $\left(2^{-\Delta C T}\right) \operatorname{method}[14]$.

\subsection{Statistical Analysis}

SPSS 25.0 software was used for statistical analysis. According to the median level of $P X N$ expression, AML patients were divided into high expression group and low expression group. Chisquare test was used to examine the relationship between $P X N$ expression and clinicopathological features. The survival curve was drawn by Kaplan-Meier method, and the long-rank test was used in the group comparison. The Cox-regression analysis was used to determine the independent prognostic factors of patients with AML. The hazard ratio (HR) and its corresponding 95\% confidence interval $(\mathrm{CI})$ were calculated. The probability value of less than 0.05 indicates statistical significance. 


\section{Results}

3.1 Up-regulation of $P X N$ expression in patients with acute myeloid leukemia

We detected the expression of $P X N$ gene in bone marrow samples of 27 patients with AML and 10 normal controls. It can be seen from Figure 1A that the expression of PXN in AML is higher than that in normal controls $(P<0.05)$, indicating that $P X N$ may be a suppressor gene in AML. In addition, we used UALCAN database to study the expression of $P X N$ gene in different FAB classification. From Figure 1B, we can see that there are differences in the expression of $P X N$ gene among different FAB classifications of leukemia, and the difference is statistically significant. Furthermore, we used GEPIA and UALCAN database to analyze the relationship between $P X N$ gene and survival in patients with AML. As shown in Figure 2, the lower expression of $P X N$ gene predicts a better prognosis of AML patients.

3.2 The relationship between the expression of $P X N$ and clinical features

Bone marrow samples from 27 patients with newly diagnosed AML were collected, and the mRNA level of $P X N$ was detected by qRT-PCR method. In order to further evaluate the clinical correlation of $P X N, 27$ patients with AML were divided into two groups according to the median: low $P X N$ expression group $(\mathrm{n}=14)$ and high $P X N$ expression group $(\mathrm{n}=13)$, as shown in Figure $3 \mathrm{~A}$. Table 1 summarized the relationship between $P X N$ expression and clinical features in 27 patients with AML, as shown in Figure 3B-F. There was no significant correlation between $P X N$ expression level and age, sex, peripheral blood leukocyte, FAB classification, treatment or not, and the mutation frequency of CEBPA, TET2, DNMT3A, NPM1 and IDH1/2.

\subsection{The prognostic value of $P X N$ expression in patients with AML}

The Kaplan-Meier method was used to evaluate the prognostic factors such as age, gene mutation, whether to receive treatment and $P X N$ expression in patients with AML. The prognosis of patients with high $P X N$ expression was poorer than that of patients with low $P X N$ expression. The Log rank test data is shown in Table 2. Multivariate regression analysis showed that refusal of treatment and high transcript level of $P X N$ were independent factors for poor prognosis in patients with AML. The survival time of AML patients with high $P X N$ expression was significantly shorter than those with low $P X N$ expression, suggesting that the prognosis of AML patients with high $P X N$ 
transcript level was poor (Table3), and the difference was statistically significant (Figure 4).

\subsection{Co-expression Network of $P X N$ in Leukemia}

We used the LinkedOmics functional module to analyze the co-expressed genes of $P X N$ in order to explore its biological significance in AML. The differentially expressed genes related to $P X N$ in AML were analyzed by Pearson Test, and the results were shown in Figure 5A. In addition, $P X N$ was positively correlated with IL27RA (Pearson correlation $=0.67, \mathrm{P}=1.141 \mathrm{E}-23$ ), CD52 (Pearson correlation $=0.59, \mathrm{P}=3.044 \mathrm{E}-17$ ) and GIMAP1 (Pearson correlation $=0.58, \mathrm{P}=1.088 \mathrm{E}-$ 16), indicating that $P X N$ is related to inflammation and immune response (Figure 5B, C).

Through the analysis of Gene Ontology (GO) database, we found that $P X N$-related coexpression genes are mainly involved in the lymphocyte-mediated immune, the positive regulation of defense response and the regulation of intercellular adhesion function (Figure 6A). Besides, through Kyoto Encyclopedia of Genes and Genomes (KEGG) analysis, it was confirmed that $P X N$ co-expression genes were enriched in cytokine receptor interaction, $\mathrm{T}$ cell receptor signal pathway, Toll-like receptor signal pathway and RAP1 signal pathway (Figure 6B). Therefore, we believe that $P X N$ has a wide range of effects on immune response and regulation in AML.

\section{Discussion}

Acute myeloid leukemia (AML) is a kind of hematopoietic malignant tumor with high heterogeneity, which is mainly characterized by uncontrolled proliferation of immature hematopoietic precursor cells in the bone marrow [1]. Now it is generally believed that the biological characteristics of AML are caused and determined by its own genetic abnormalities [14]. In recent years, great progress has been made in the search for prognostic markers of AML at the genetic level and its application in the re-stratification of AML. The screening for novel molecular markers with gene mutation or abnormal expression will further update people's understanding of the pathogenesis of AML [15].

$P X N$ is located on human chromosome $12 \mathrm{q} 24.31$ and encodes a cytoskeleton protein that involves the adhesion of actin membrane to extracellular matrix $[15,16]$. The high expression of $P X N$ in some tumors is related to tumor stage, poor differentiation, lymphatic metastasis, distant metastasis and tumor lymph node metastasis stage $[17,18]$. However, the relationship between $P X N$ and AML has not been reported before. Therefore, we collected AML and normal samples to explore 
the expression and prognostic value of $P X N$ mRNA in AML.

In this study, GEPIA database analysis showed that the level of $P X N$ transcription in AML patients was higher than that in normal subjects, and the prognosis of patients with high expression of $P X N$ was poor. Subsequently, we collected bone marrow samples from 49 newly diagnosed AML patients for qRT-PCR detection, and reached the same conclusion. Through Kaplan-Meier curve and log rank test, we found that the high expression of $P X N$ was significantly correlated with OS in patients with AML, which was consistent with the results of GEPIA and UALCAN database. Therefore, we think that $P X N$ can be used as a prognostic marker for AML patients.

In order to further explore the biological significance of $P X N$ in AML, we used LinkedOmics functional module to analyze $P X N$ co-expression genes. The results show that $P X N$ participates in a variety of functional networks related to inflammation and immune response, such as lymphocytemediated immunity, positive regulation of defense response, $\mathrm{T}$ cell receptor and Toll-like receptor signal pathway.

$P X N$ is an important component of the focal adhesion complex, which is related to the occurrence, development and metastasis of human cancer, and is related to the poor clinical prognosis of tumor patients, which has been reported in many previous studies[19-21]. $P X N$ is directly regulated by ETV4, and its overexpression is related to the poor prognosis of human NSCLC[22]. $P X N$ promotes tumor progression and metastasis of gastric cancer, breast cancer and colorectal cancer, and its overexpression is associated with poor prognosis [15, 23-25]. CD8+T cells play an important role in immune-related tolerance and immunosuppression in tumor microenvironment (TME)[26]. It has been reported that in breast cancer, lung squamous cell carcinoma and cutaneous melanoma, the expression of $P X N$ is negatively correlated with the infiltration of $\mathrm{CD} 8+$ and human epidermal growth factor receptor 2-negative $\mathrm{T}$ cells, while the expression of $P X N$ in DLBC is positively correlated with CD8+T cell infiltration[27]. The binding of $P X N$ and $\mathrm{CD} 103$ triggers the signal transduction of $\alpha_{\mathrm{E}} \beta_{7}$ integrin from outside to inside, which promotes the migration behavior and effect function of CD8+T cells[28]. Other studies have shown that $P X N$ has an important relationship with macrophages and phagocytosis[29]. In macrophages, miR-375 targets $P X N$, directly to enhance the migration and infiltration of macrophages to tumor spheroids and xenografted mice[30]. Taken together, $P X N$ has a wide range of effects on immune response, and we speculate that the poor prognosis caused by the high expression of $P X N$ in AML 
may be related to the immune infiltration and immune escape of tumor cells.

In conclusion, our research shows that the expression of $P X N$ in AML patients is higher than that in healthy people, and the AML patients with high expression of $P X N$ indicate a poor prognosis. This is the first report of $P X N$ in AML. Our group is expanding the sample size, hoping to further study the signal transduction pathway between the high expression of PXN and AML.

\section{Conclusions}

Our research shows that the expression of $P X N$ in AML patients is higher than that in healthy people, and the AML patients with high expression of $P X N$ indicate a poor prognosis. This is the first report of $P X N$ in AML. Our group is expanding the sample size, hoping to further study the signal transduction pathway between the high expression of PXN and AML.

\section{Supplementary information}

\section{Acknowledgements}

This study was supported by Sichuan department of science and technology (grant no. 20YYJC0940) and Luzhou people's government-Southwest Medical University joint project (grant no. 2017LZXNYD-Z06)

\section{Authors' contributions}

CONCEPTION: Chunlan Huang, Xinwen Zhang, Hao Xiong

INTERPRETA TION OR ANAL YSIS OF DA TA: Xinwen Zhang, Hao Xiong, Jialin Duan, Xiaomin Chen, Yang Liu

PREPARA TION OF THE MANUSCRIPT: Xinwen Zhang, Hao Xiong

REVISION FOR IMPORTANT INTELLECTUAL CONTENT: Xinwen Zhang, Hao Xiong, Jialin Duan, Xiaomin, Chen, Yang Liu

SUPERVISION: Chunlan, Huang

\section{Funding}

This study was not supported by any funding. 


\section{Ethics approval and consent to participate}

Research Involving Human Participants and/or Animals: This study involves blood and bone marrow aspirates from AML patients. Ethical approval was obtained from institutional ethics committee (AF-V4-22.0) to conduct the study.

Informed Consent: Informed consent was obtained from all individual participants included in the study.

Ethical approval: All procedures performed in studies involving human participants were in accordance with the ethical standards of the institutional committee.

\section{Consent for publication}

Not Applicable.

\section{Competing interests}

The authors declare that they have no competing interests.

\section{References}

1. Papaemmanuil E, Gerstung M, Bullinger L, et al. Genomic Classification and Prognosis in Acute Myeloid Leukemia. N Eng1 J Med. 2016; 374:2209-21.

2. $\mathrm{Xu} \mathrm{XH}$, Zhang L, Cao XX, et al. Evaluation of the implementation rate of primary antifungal prophylaxis and the prognosis of invasive fungal disease in acute leukemia patients in China. J Infect Chemother. 2017; 23:360-7.

3. Slovak ML, Kopecky KJ, Cassileth PA, et al. Karyotypic analysis predicts outcome of preremission and postremission therapy in adult acute myeloid leukemia: a Southwest Oncology Group/Eastern Cooperative Oncology Group Study. Blood. 2000; 96:4075-83.

4. Wang $\mathrm{M}$, Lindberg $\mathrm{J}$, Klevebring $\mathrm{D}$, et al. Validation of risk stratification models in acute myeloid leukemia using sequencing-based molecular profiling. Leukemia. 2017; 31:2029-36.

5. Kawada I, Hasina R, Lennon FE, et al. Paxillin mutations affect focal adhesions and lead to 
altered mitochondrial dynamics: relevance to lung cancer. Cancer Biol Ther. 2013; 14:679-91.

6. Turner CE. Paxillin. Int J Biochem Cell Biol. 1998; 30:955-9.

7. López-Colomé AM, Lee-Rivera I, Benavides-Hidalgo R, López E. Paxillin: a crossroad in pathological cell migration. J Hematol Oncol. 2017; 10:50.

8. Ketscher A, Jilg CA, Willmann D, et al. LSD1 controls metastasis of androgen-independent prostate cancer cells through PXN and LPAR6. Oncogenesis. 2014; 3: e120.

9. Chen DL, Wang DS, Wu WJ, et al. Overexpression of paxillin induced by miR-137 suppression promotes tumor progression and metastasis in colorectal cancer. Carcinogenesis. 2013; 34:80311.

10. Tang Z, Li C, Kang B, Gao G, Li C, Zhang Z. GEPIA: a web server for cancer and normal gene expression profiling and interactive analyses. Nucleic Acids Res. 2017; 45: W98-W102.

11. Chandrashekar DS, Bashel B, Balasubramanya SAH, et al. UALCAN: A Portal for Facilitating Tumor Subgroup Gene Expression and Survival Analyses. Neoplasia. 2017; 19:649-58.

12. Vasaikar SV, Straub P, Wang J, Zhang B. LinkedOmics: analyzing multi-omics data within and across 32 cancer types. Nucleic Acids Res. 2018; 46: D956-63.

13. Vardiman JW, Thiele J, Arber DA, et al. The 2008 revision of the World Health Organization (WHO) classification of myeloid neoplasms and acute leukemia: rationale and important changes. Blood. 2009; 114:937-51.

14. Pan K, Liang XT, Zhang HK, et al. Characterization of bridging integrator 1 (BIN1) as a potential tumor suppressor and prognostic marker in hepatocellular carcinoma. Mol Med. 2012; 18:507-18.

15. Chen DL, Wang ZQ, Ren C, et al. Abnormal expression of paxillin correlates with tumor progression and poor survival in patients with gastric cancer. J Transl Med. 2013; 11:277.

16. Turner CE. Paxillin and focal adhesion signalling. Nat Cell Biol. 2000; 2: E231-6.

17. Wen L, Zhang $\mathrm{X}$, Zhang $\mathrm{J}$, et al. Paxillin knockdown suppresses metastasis and epithelial-mesenchymal transition in colorectal cancer via the ERK signalling pathway. Oncol Rep. 2020; 44:1105-15.

18. Liu Q, Wang J, Tang M, et al. The overexpression of PXN promotes tumor progression and leads to radioresistance in cervical cancer. Future Oncol. 2018; 14:241-53.

19. McLean GW, Carragher NO, Avizienyte E, Evans J, Brunton VG, Frame MC. The role of 
focal-adhesion kinase in cancer - a new therapeutic opportunity. Nat Rev Cancer. 2005; 5:50515.

20. Noh K, Bach DH, Choi HJ, et al. The hidden role of paxillin: localization to nucleus promotes tumor angiogenesis. Oncogene. 2021; 40:384-95

21. Schaller MD. FAK and paxillin: regulators of N-cadherin adhesion and inhibitors of cell migration? J Cell Biol. 2004; 166:157-9.

22. Wang Y, Ding X, Liu B, et al. ETV4 overexpression promotes progression of non-small cell lung cancer by upregulating PXN and MMP1 transcriptionally. Mol Carcinog. 2020; 59:73-86.

23. Deakin NO, Turner CE. Distinct roles for paxillin and Hic-5 in regulating breast cancer cell morphology, invasion, and metastasis. Mol Biol Cell. 2011; 22:327-41.

24. Tabassam FH, Graham DY, Yamaoka Y. Paxillin is a novel cellular target for converging Helicobacter pylori-induced cellular signaling. Am J Physiol Gastrointest Liver Physiol. 2011; 301: G601-11

25. Li D, Ding J, Wang X, Wang C, Wu T. Fibronectin promotes tyrosine phosphorylation of paxillin and cell invasiveness in the gastric cancer cell line AGS. Tumori. 2009; 95:769-79.

26. Iwahori K. Cytotoxic CD8+ Lymphocytes in the Tumor Microenvironment. Adv Exp Med Biol. 2020; 1224:53-62.

27. Chen Y, Zhao H, Xiao Y, et al. Pan-cancer analysis reveals an immunological role and prognostic potential of PXN in human cancer. Aging (Albany NY). 2021; 13.

28. Gauthier L, Corgnac S, Boutet M, et al. Paxillin Binding to the Cytoplasmic Domain of CD103 Promotes Cell Adhesion and Effector Functions for CD8+ Resident Memory T Cells in Tumors Cancer Res. 2017; 77:7072-82.

29. Liu H, Zhu L, Dudiki T, et al. Macrophage Migration and Phagocytosis Are Controlled by Kindlin-3's Link to the Cytoskeleton. J Immunol. 2020; 204:1954-67.

30. Frank AC, Ebersberger S, Fink AF, et al. Apoptotic tumor cell-derived microRNA-375 uses CD36 to alter the tumor-associated macrophage phenotype. Nat Commun. 2019; 10:1135. 
A

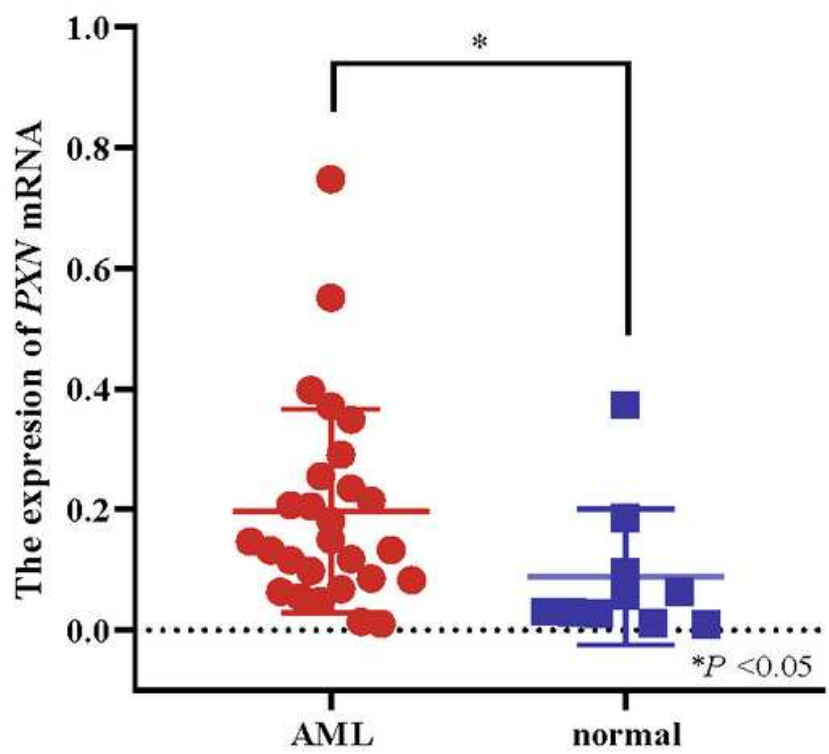

B

Expression of PXN in LAML based on French American British classification

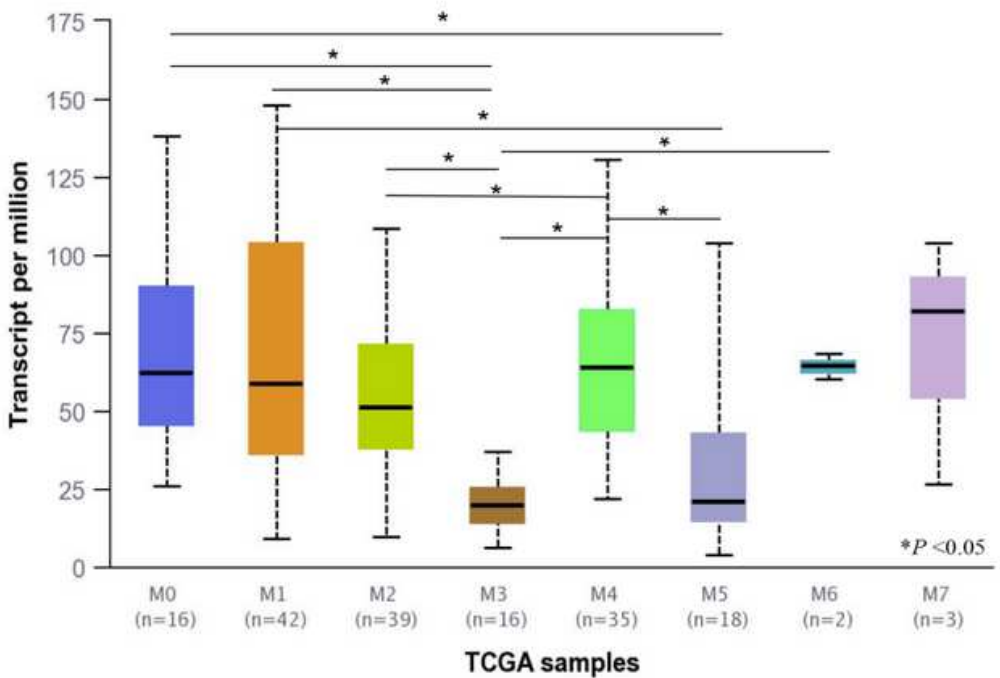

\section{Figure 1}

The expression levels of PXN based on common data base. A) The expression levels of PXN in AML patients $(n=27)$ and Normal $(n=10)$. B) The expression levels of PXN in AML based on FAB classification (UALCAN).
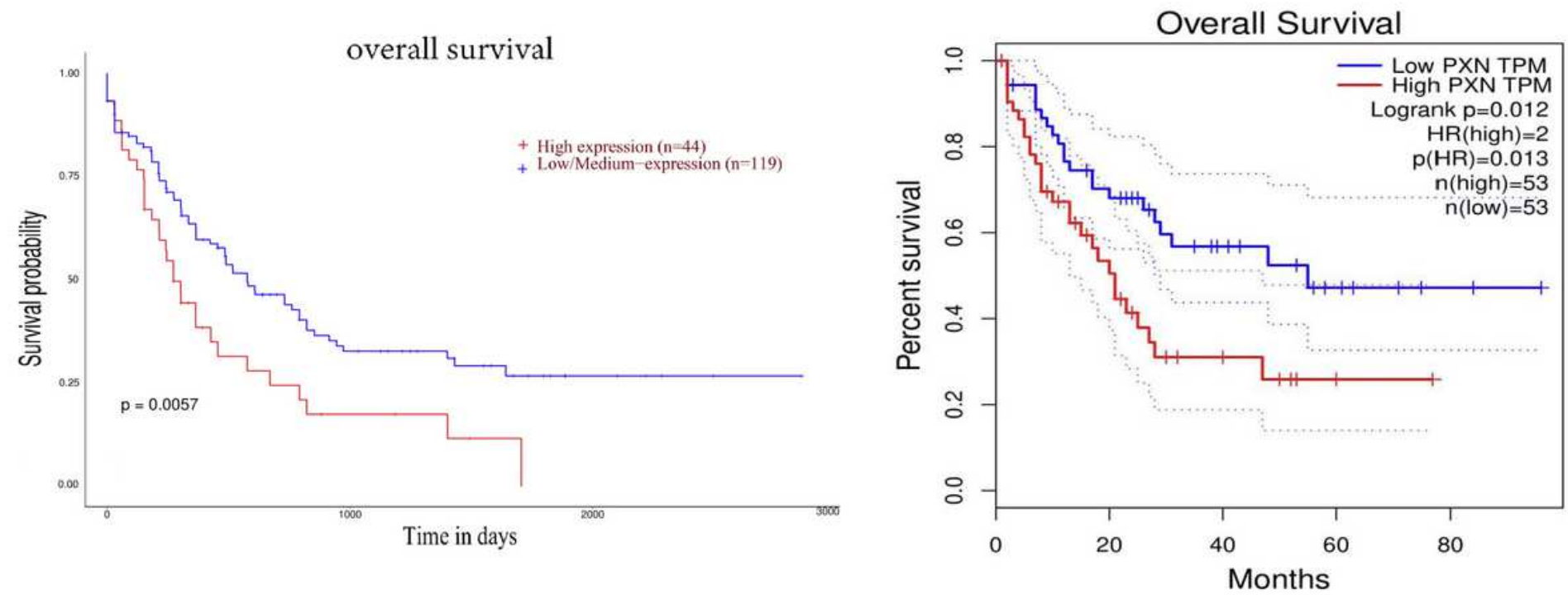

Figure 2

The effect of PXN expression level on the survival outcomes of patients was analyzed based on datasets. A): The relationship between PXN expression and prognosis in patients with AML based on UALCAN 
dataset. B): The relationship between PXN expression and prognosis of patients with AML based on GEPIA dataset.
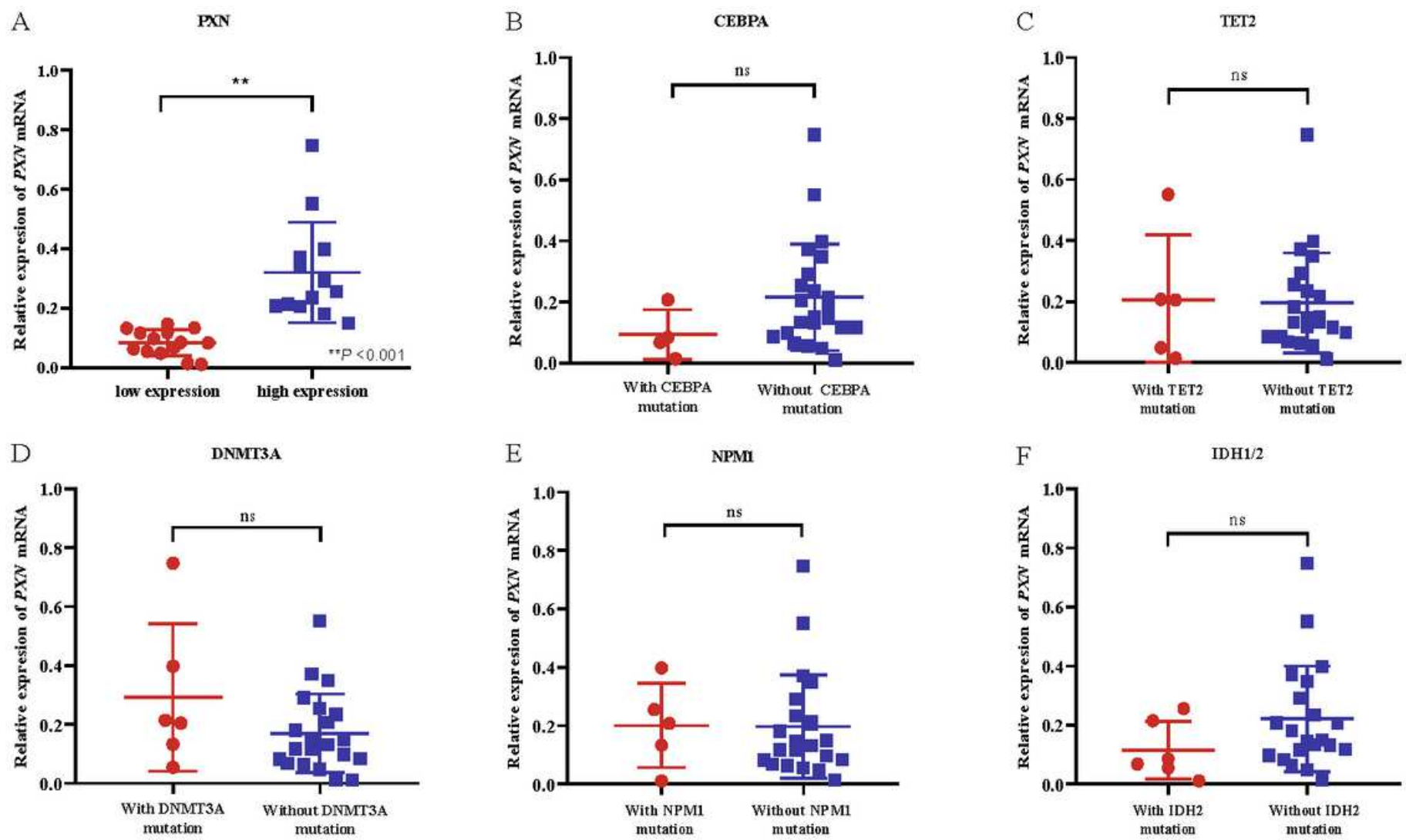

\section{Figure 3}

The relationship between the expression level of PXN and clinicopathological characteristics. A) AML patients were divided into high expression group and low expression group according to the median expression of PXN. B-F) The correlation between PXN expression level and CEBPADTET2 $2 D N M T A 3 \square$ NPM1 and IDH1/2 gene mutation. 
PXN

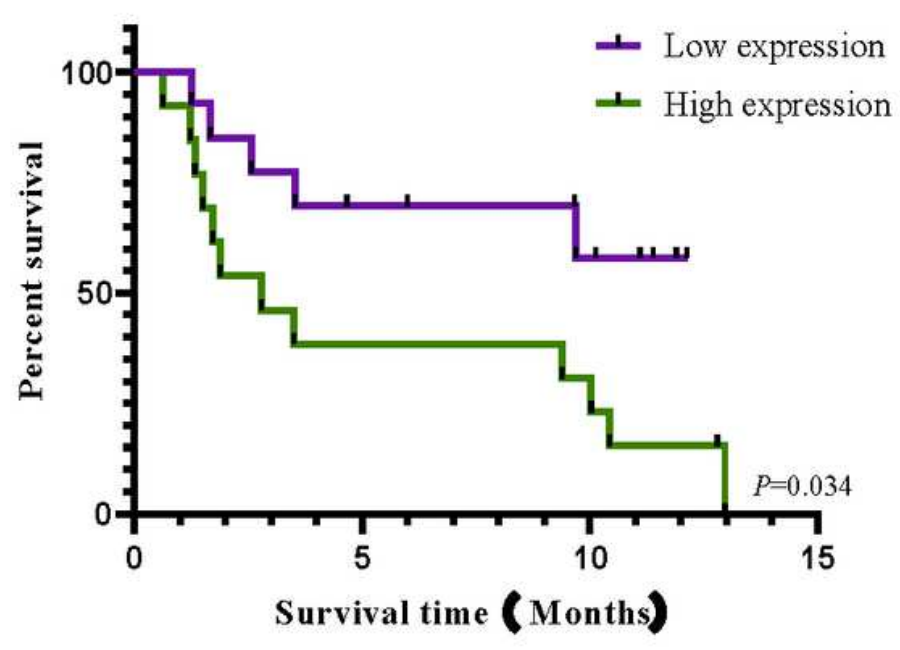

treatment condition

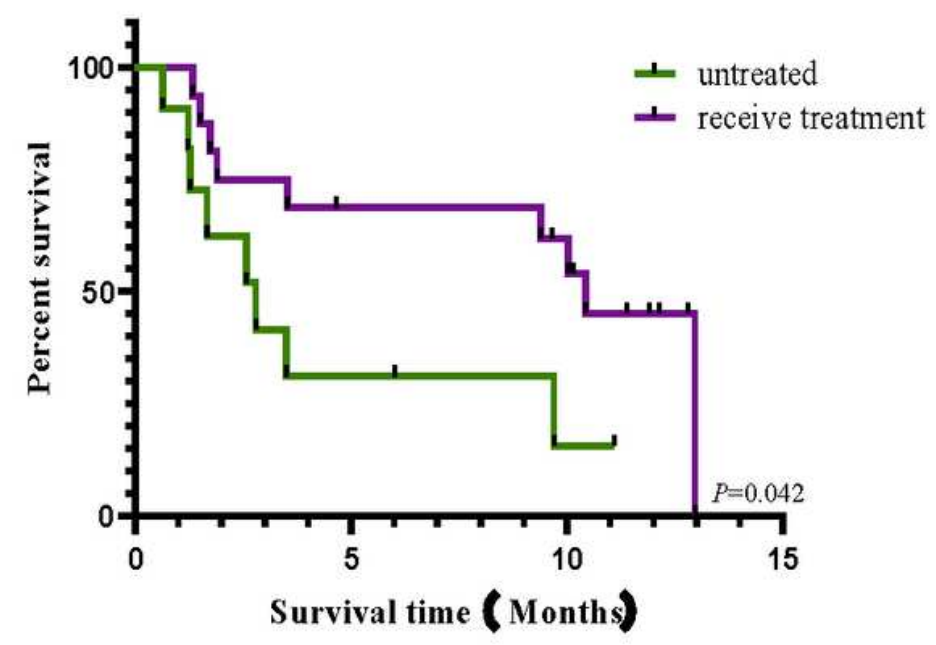

\section{Figure 4}

The Kaplan-Meier survival analysis was used to analyze the prognostic factors of 27 AML patients. A) The relationship between PXN expression and overall survival time in 27 AML patients. B) The relationship between treatment condition and overall survival time in 27 AML patients. 
A

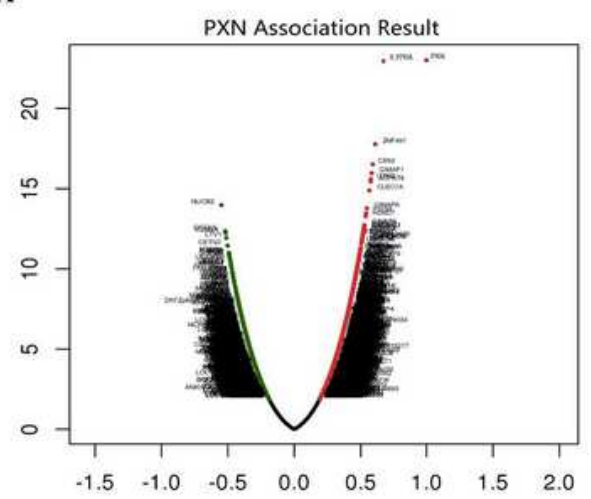

Pearson Correlation Coefficient (Pearson test)

B

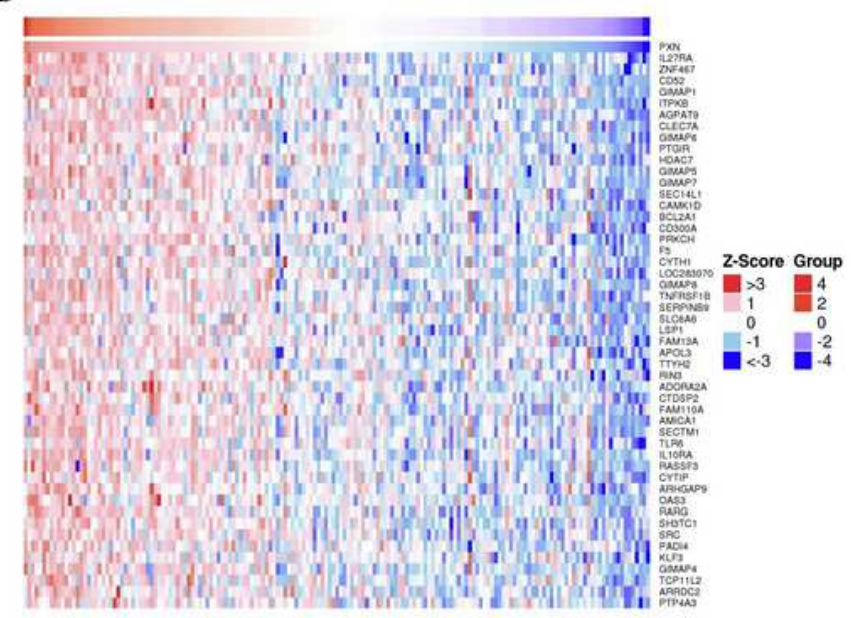

C

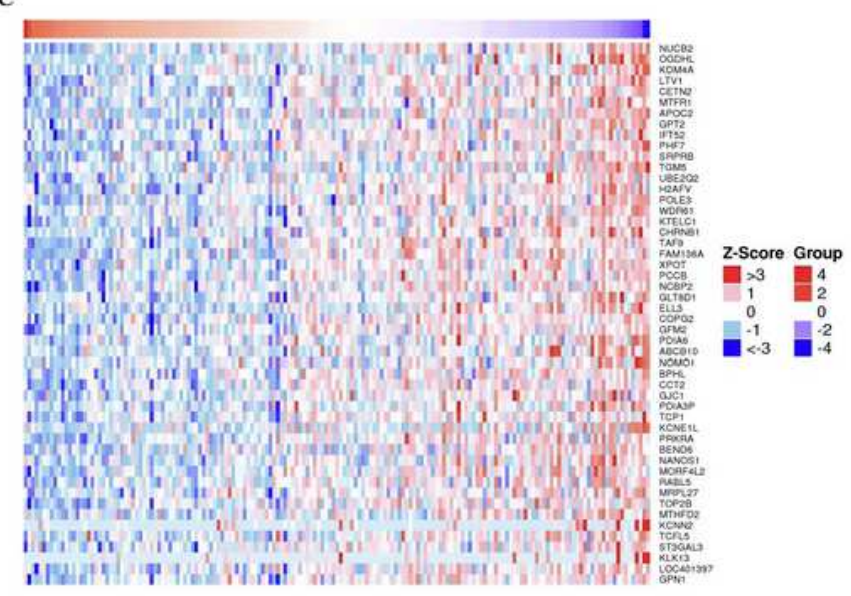

\section{Figure 5}

The differentially expressed genes related to PAW in AML (LinkedOmics). (A) Using Pearson test to analyze genes differentially expressed in correlation with PXN in AML.(B) Heat map shows the top 50 genes with significant positive correlation with PXN. (C) Heat map showed that the top 50 genes were significantly negatively correlated with PXN. 
A

GO-BP

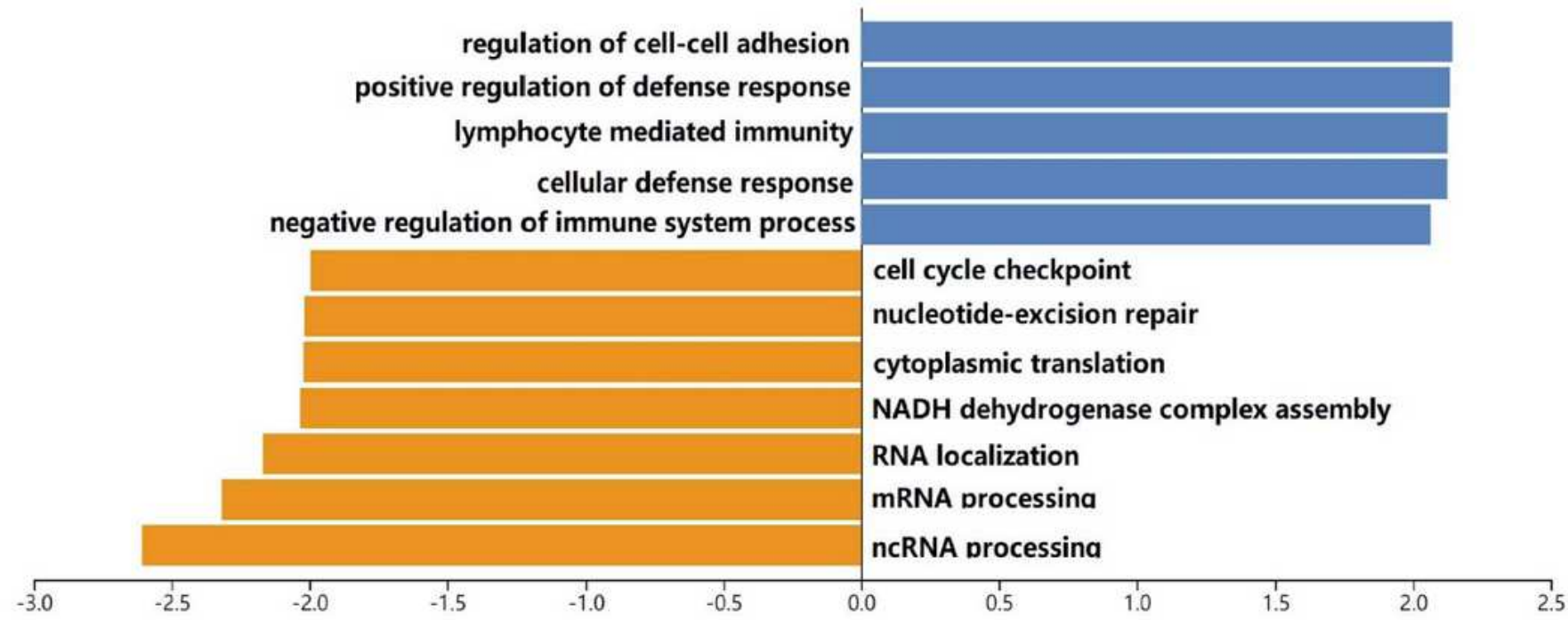

Normalized Enrichment Score

$\mathrm{B}$

FDR $\leq 0.05 \square$ FDR $>0.05$

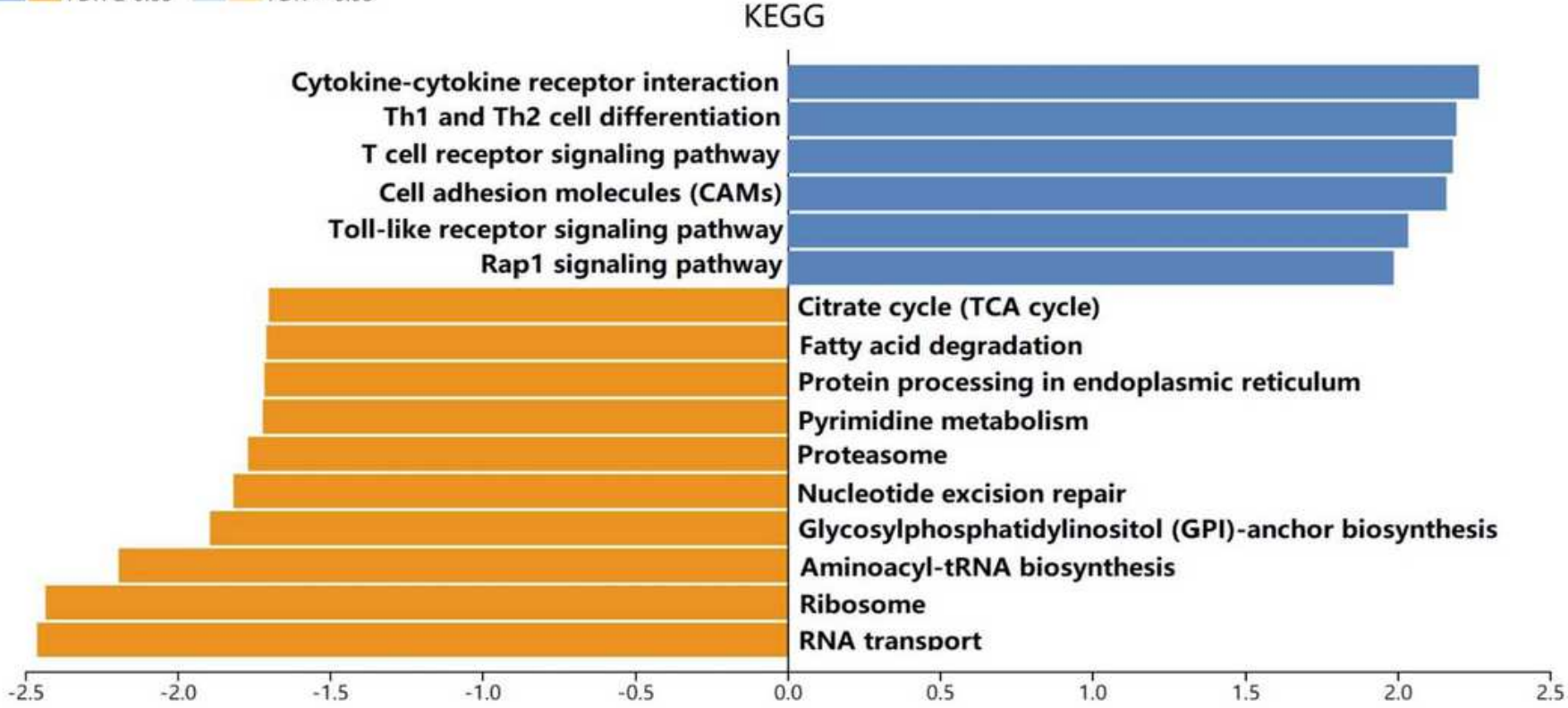

Normalized Enrichment Score

Figure 6

GO annotations and KEGG pathways enriched with PXN co-expressed genes in leukemia (LinkedOmics). Using GSEA to analyze the significantly enriched GO-BP and KEGG pathways of PXN co-expression genes in AML. (A) Gene Ontology Biological processes, analyzed by GSEA. (B) KEGG pathway, analyzed by GSEA The Leading EdgeNum and the false discovery rate (FOR) are shown in blue and orange column, respectively. 


\section{Supplementary Files}

This is a list of supplementary files associated with this preprint. Click to download.

- renameda76a0.pdf 